\title{
Implicaciones en la salud de los $\beta$-glucanos de cebada
}

\author{
Health implications of barley $\beta$-glucans \\ Amalfi M. García-Luna ${ }^{a}$, Ernesto Alanís-García ${ }^{b}$
}

\begin{abstract}
:
Barley grain is an important source of $\beta$-glucan, which is a polysaccharide that is part of soluble dietary fiber (DF). Soluble fiber has better hydration properties, as does fermentation and the production of short chain fatty acids (SCFA). $\beta$-glucans provide numerous benefits to human health. The objective of this review is to analyze the current evidence and report on the modifications it undergoes during consumption, its addition and functional properties of barley $\beta$-glucans, allowing to obtain the health benefits (hypolipidemic, hypoglycemic and hypocholesterolemic). According to the review, they have been found to help lower total cholesterol and LDL-C, prevent visceral fat and the risk of diverticular disease, as well as cause delayed gastric emptying and attenuation of the absorption rate of glucose in the small intestine. They can also help relieve gastric lesions and participate in anticancer activity and lung carcinoma; also, its topical application improves the healing of diabetic and venous ulcers. Evidence from recent studies consistently shows that $\beta$-glucan consumption is associated with multiple benefits, from decreased macronutrients absorption to wound healing. This further highlights the importance of its consumption and application.
\end{abstract}

Keywords:

Barley, $\beta$-glucan, dietary fiber, health

Resumen:

El grano de cebada es una importante fuente de $\beta$-glucanos, el cual es un polisacárido que forma parte de la fibra dietética (FD) soluble. La fibra soluble tiene mejores propiedades de hidratación, al igual que la fermentación y la producción de ácidos grasos de cadena corta (AGCC). Los $\beta$-glucanos proporcionan numerosos beneficios a la salud humana. La presente revisión tiene como objetivo analizar la evidencia actual e informar sobre las modificaciones que sufre durante el consumo, su adición y propiedades funcionales de los $\beta$-glucanos de cebada, permitiendo obtener los beneficios a la salud (hipolipidémico, hipoglucémico e hipocolesterolémico). De acuerdo con la revisión, se ha encontrado que contribuyen a disminuir el colesterol total y el cLDL, a prevenir la grasa visceral y el riesgo de enfermedad diverticular, así mismo provocan retraso en el vaciamiento gástrico y atenuación de la velocidad de absorción de la glucosa en el intestino delgado. Además, pueden colaborar a aliviar lesiones gástricas y participar en la actividad anticancerosa y el carcinoma de pulmón; también, su aplicación tópica mejora la cicatrización de úlceras diabéticas y venosas. La evidencia de los estudios recientes demuestra consistentemente que el consumo de $\beta$-glucanos se asocia con múltiples beneficios, desde la disminución de la absorción de macronutrientes, hasta la cicatrización de heridas. Esto destaca aún más la importancia de su consumo y utilización.

Palabras Clave:

Cebada, $\beta$-glucanos, fibra dietética, salud

\section{Introducción}

A los $\beta$-glucanos se les pueden adjudicar múltiples beneficios a la salud humana, dentro de los que se encuentran, la reducción en el contenido o la digestibilidad de macronutrientes de los alimentos naturales o procesados, así como efectos hipolipidémicos, hipoglucémicos e hipocolesterolémicos

\footnotetext{
${ }^{a}$ Universidad Autónoma del Estado de Hidalgo, https://orcid.org/0000-0002-8975-2334, Email: montseeluuna97586@gmail.com b Autor de Correspondencia, Universidad Autónoma del Estado de Hidalgo, https://orcid.org/0000-0003-1540-4908, Email:
} ernesto_alanis@uaeh.edu.mx 
1. La Administración de Alimentos y Medicamentos de Estados Unidos (FDA por sus siglas en inglés), indica que el consumo diario, en la dieta, de $3 \mathrm{~g}$ de $\beta$-glucanos de cebada son suficientes para observar un decremento de los niveles de colesterol sérico total y de lipoproteínas de baja densidad $(L D L)^{2}$. Se ha demostrado, de igual forma, que los $\beta$-glucanos reducen la glucosa postprandial debido a que aumentan la viscosidad en el tracto digestivo superior y brindan una sensación de saciedad ${ }^{3}$. Estos $\beta$-glucanos (también conocidos como glucanos de enlace mixto) son fibras dietéticas solubles fácilmente disponibles en cereales como avena y cebada, distribuidos tanto en el endospermo como en las capas de aleurona ${ }^{3,4}$. Por lo que en los últimos años han estado ganando interés debido a sus múltiples funciones y propiedades bioactivas ${ }^{4}$. Además, la cebada (Hordeum vulgare L.), como fuente importante de $\beta$-glucanos, al incorporarse en productos a base de cereales contribuye a la ingesta de otros compuestos bioactivos como los fenólicos ${ }^{5}$. Este cereal es un antiguo e importante cultivo, utilizado por primera vez como grano para la alimentación humana, pero con el paso del tiempo se transformó en grano malteado y en cerveza principalmente ${ }^{6,7}$. En la actualidad, la cebada ocupa el quinto lugar con mayor producción a escala mundial (152 millones de ton). Solo la Unión Europa producen 62 millones de toneladas al año con el $50 \%$ del área y representando más del $39 \%$ del volumen de producción mundial. En México, la producción fue estimada en un millón de toneladas al 2020, concentrando más del $89 \%$ los estados de Guanajuato, Hidalgo, Tlaxcala, Puebla, México y Zacatecas ${ }^{8}$.

El objetivo de esta revisión ha sido analizar la evidencia actual e informar sobre las modificaciones que sufre durante el consumo, su adición y propiedades funcionales de los $\beta$-glucanos de cebada, permitiendo obtener los beneficios a la salud.

\section{Generalidades sobre fibra dietética}

Tradicionalmente, la idea del concepto de fibra dietética (FD) abarca a todos aquellos hidratos de carbono que no se digieren ni absorben en la parte alta del tubo intestinal, por lo tanto, llegan intactos al colon ${ }^{9,10}$. En consecuencia, se trata de una entidad heterogénea que engloba un número importante de distintos compuestos. Por sus características fisiológicas y múltiples efectos a nivel del tránsito digestivo se considera que tiene un papel importante en la fisiopatología, la prevención y el tratamiento de múltiples enfermedades ${ }^{9}$. Así, el consumo alto de FD se ha asociado con una reducción en el riesgo de padecer enfermedad diverticular, sugiriendo que el consumo, en la dieta, de $30 \mathrm{~g}$ de fibra por día contribuye a reducir en un $41 \%$ el riesgo de padecer tal enfermedad, en comparación con las personas con una baja ingesta de fibra ${ }^{10}$. También, este consumo reduce entre un 10 $20 \%$ las LDL, teniendo un beneficio a la salud cardiovascular ${ }^{3}$.

La FD, que se encuentra en los cereales, es un grupo de carbohidratos complejos con diez o más unidades monoméricas; conformados por celulosa, hemicelulosa, lignina, gomas, mucílago, oligosacáridos, pectina, $\beta$ glucanos y otras sustancias menores asociadas, cómo ceras, cutina y suberina. Sus diferencias estructurales determinan sus propiedades físicas, químicas y fisiológicas, y dependiendo de su composición se pueden clasificar en carbohidratos no digeribles, cómo fibra cruda, polisacárido no amiláceos, FD soluble, FD insoluble, fracción no digerible y almidón resistente ${ }^{3}$. Además de los constituyentes mencionados, para poder diferenciar el tipo de fibra se deben aplicar otros criterios como la composición química, la ubicación en la pared celular y la solubilidad de estas macromoléculas, para así poder situar en alguno de los tipos de fibra, existiendo FD soluble como pectinas, mucílagos y ciertos tipos de hemicelulosas; o FD insoluble como celulosa y hemicelulosas ${ }^{11}$.

Cada uno de los tipos de fibra efectúa funciones distintas en el organismo, por ejemplo, las fibras solubles tienen mejores propiedades de hidratación que dan como resultado la característica viscosidad, al igual que la fermentación, y la producción de ácidos grasos de cadena corta (AGCC) que estimulan la producción de las hormonas péptido similar al glucagón tipo 1 (GLP1) y péptido YY (PYY), las cuales son hormonas que regulan el apetito y la saciedad, por lo que resulta un mecanismo importante para la modulación del sobrepeso y la obesidad ${ }^{13}$. Por otro lado, las fibras insolubles están relacionadas con el incremento de la masa y el volumen fecal, la reducción del tiempo de tránsito intestinal, disminuyendo la posibilidad de estreñimiento y cáncer de colon ${ }^{12,13}$.

En el transcurso del tiempo se han evidenciado efectos físicos de la FD, entre ellos, que la ingesta de alimentos con alto contenido de fibra induce a la prolongación del tiempo de masticado, permitiendo la secreción de una mayor cantidad de saliva y de jugos gástricos, resultando en una expansión del estómago e incremento de la saciedad ${ }^{13}$. De acuerdo con Wong (2006), solo las fibras viscosas solubles (ej. pectina, $\beta$-glucanos, psyllium, metilcelulosa) retrasan el tiempo de vaciado gástrico y absorción lenta de glucosa, en tanto que las fibras solubles no viscosas (ej. inulina, dextrinas) actúan primeramente como sustrato para fermentación microbiana en el colon ${ }^{14}$. 


\section{Características de los $\beta$-glucanos}

Los $\beta$-glucanos son polisacáridos lineales de unidades de glucosa conformados de residuos consecutivos de $\beta-D$ glucopiranosilo unidos por enlaces $1 \rightarrow 4$ separados por enlaces simples $1 \rightarrow 3$ (figura 1 ), de elevado peso molecular (530 a 2,210 kDa) ${ }^{15}$.

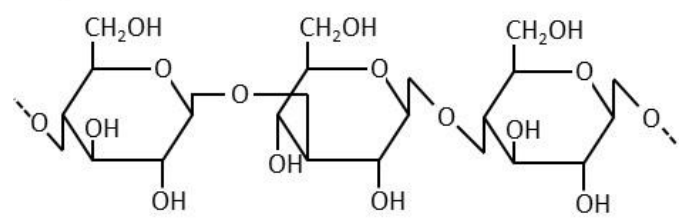

Figura 1. Estructura molecular de $\beta$-glucanos de enlaces mixtos $(1 \rightarrow 3)(1 \rightarrow 4)$

En la cebada este polisacárido se localiza en las paredes de células de endospermo y aleurona. Constituye del 2 al $11 \%$ del peso del total de carbohidratos del grano, aunque regularmente oscila entre 4 y $7 \%$. No obstante, es difícil extraer $\beta$-glucanos, debido a que se encuentran acomplejados con el almidón, en la matriz proteica y con lípidos en el grano. Sin embargo, este es regulado por la enzima $\beta$-glucano endohidrolasa $(1 \rightarrow 3,1 \rightarrow 4)$, conocida de otra forma como licheninasa $o \quad 1 \rightarrow 3,1 \rightarrow 4 \quad \beta$ glucanasa, cuya función es promover la degradación de la pared celular del endospermo durante la germinación 6,16 .

\section{Consumo de cebada}

La cebada es considerada un cultivo experimental modelo, en virtud de su corta vida útil y sus características morfológicas, fisiológicas y genéticas. Las evidencias arqueológicas indican que la cebada fue más importante que el trigo en las civilizaciones basadas en la agricultura y se cultivó principalmente para consumo humano ${ }^{17}$.

Es un cultivo adaptado a grandes altitudes y soporta, en mayor medida, las tensiones abióticas de la zona en comparación con otros cultivos. La cebada se produce comúnmente por encima de los 4,000 m sobre el nivel del mar. Su precocidad y tolerancia al frío la hace viable para las temporadas de crecimiento cortas y sin heladas en las grandes altitudes de los Andes. Los principales usos del cultivo son para el consumo humano, pastoreo directo de pequeños rumiantes, producción de heno $\mathrm{y}$, a veces, ensilaje ${ }^{18}$. Se considera predominantemente como cultivo alimenticio en muchas partes del mundo como las regiones semiáridas de África, Medio Oriente, tierras altas de Nepal, Etiopía y Tíbet, países andinos de América del Sur y en algunos países asiáticos y el Himalaya ${ }^{17}$.

Como se muestra en la tabla 1 , el grano de cebada contiene aproximadamente de 4 a $9 \%$ de $\beta$-glucanos. Además, por cada $100 \mathrm{~g}$ de peso seco de cebada, se ha reportado de 2 a $20 \mathrm{~g}$ de $\beta$-glucanos, de los cuales $65 \%$ son hidrosoluble. Además, son una fuente importante en tocoles, que incluye tocoferoles y tocotrienoles, mismos que disminuyen el colesterol LDL sérico a través de su acción antioxidante ${ }^{16,19}$.

Tabla 1. Cantidades porcentuales de la composición del grano de cebada $^{19}$

\begin{tabular}{|l|c|}
\hline \multicolumn{2}{|c|}{$\begin{array}{c}\text { Composición del grano entero de } \\
\text { cebada (\%) en base seca }\end{array}$} \\
\hline Almidón & $65-68$ \\
\hline Proteína & $10-17$ \\
\hline Lípidos & $2-3$ \\
\hline Minerales & $1.5-2.5$ \\
\hline FD total & $11-34$ \\
\hline FD soluble & $3-20$ \\
\hline$\beta$-glucanos & $4-9$ \\
\hline
\end{tabular}

Anteriormente, el consumo de cebada era sobre todo mediante la cerveza. La primera cervecería mexicana comenzó en 1542 en la Nueva España y se llamó "Brazería" 7 . Con el paso de los años, en México surgió la necesidad de cultivar cebada, para elaborar la cerveza y disminuir los gastos excesivos de materia prima. Actualmente México se coloca como el primer exportador de cerveza y el cuarto productor de cerveza a escala mundial $^{20}$.

\section{Cambios durante la digestión}

Se han publicado una serie de trabajos en los últimos años sobre el efecto del consumo y usos de $\beta$-glucanos de cebada, donde se ha comprobado que tienen ventajas para la salud, algunas de ellas son atribuidas a la formación de soluciones viscosas, lo cual provoca un retraso en el vaciamiento gástrico, que como consecuencia interfiere en los procesos de digestión y absorción de los nutrientes, por lo que parece ser un determinante importante sobre los parámetros lipídicos y la reducción del índice glicémico, entre otros beneficios $3,16,30$. Por lo anterior, se han aplicado diferentes tratamientos enzimáticos para liberar una mayor cantidad de $\beta$-glucanos, obteniendo extractos con diferente pureza, peso molecular y comportamiento reológico. Concluyen que durante los procesos de extracción y purificación es inevitable la pérdida de viscosidad de las preparaciones de $\beta$-glucanos, debido a la degradación y reducción en el peso molecular, aunque esto no es deseable por sus beneficios para la salud ${ }^{4,21}$.

Se ha comprobado que la digestibilidad del almidón puede ser influenciado por la estructura del almidón, el cultivar, el contenido de $\beta$-glucanos y la sensibilidad de la enzima. Así mismo, se demostró que para aumentar considerablemente la digestibilidad del almidón se debe 
calentar la harina de cebada ${ }^{6}$. Para poder hidrolizar los $\beta$-glucanos es necesario de la presencia de una enzima llamada glucanasa, la cual puede degradar los $\beta$ glucanos de alto peso molecular a bajo peso molecular. Los fragmentos de oligosacáridos, que disminuyen la viscosidad de los $\beta$-glucanos, fomentan la reticulación entre las moléculas de gluten e indirectamente mejora la estructura de la red de gluten ${ }^{22}$.

Desde otra perspectiva, Danilova y colaboradores (2017) demostraron un aumento importante de $\beta$-glucanos, por medio de translocaciones Robertsonianas de brazo completo (RobT) que involucran el cromosoma $7 \mathrm{H}$ de cebada y tres cromosomas de trigo del grupo 7, junto con un recurso de reservas genéticas que probablemente transporten muchos otros genes importantes de la cebada al trigo ${ }^{23}$. Sin embargo, se pueden ver afectadas sus propiedades fisicoquímicas y funcionales a causa de su proceso de extracción.

Por lo anterior, Zhao y colaboradores (2020) reportaron un nuevo método que redujo el tiempo de extracción a la mitad y aumentó el rendimiento aproximadamente un $10 \%$. Consiste en un tratamiento con microfluidización dinámica de alta presión (DHPM), la cuál es una tecnología que integra operaciones de unidades múltiples como mezcla, trituración, homogeneización y transporte, a pesar de ello, puede perjudicar la microestructura de los materiales por impacto, cizallamiento a alta velocidad, liberación de presión instantánea y formación estructuras porosas. Ésta redujo la viscosidad de $\beta$-glucanos de cebada, así como también la distribución del tamaño de partícula de agregación. Aunque el peso molecular se redujo por el tratamiento con DHPM, el tratamiento físico exógeno no cambió la estructura primaria ${ }^{24}$.

\section{Evidencias sobre la salud}

Existen diversas patologías que afectan a un gran número de personas, sin embargo, a través del tiempo, múltiples investigadores han llevado a cabo exploraciones para poder aliviar o disminuir sintomatologías que traen consigo éstas. Actualmente ya se cuenta con referencias que muestran que los $\beta$ glucanos son benéficos para la salud de los seres humanos. Los $\beta$-glucanos tienen un amplio espectro de efectos sobre distintos tipos de células. Se ha demostrado que el consumo de $3 \mathrm{~g}$ o más de $\beta$-glucanos al día, disminuye significativamente los niveles de LDL y colesterol total, en $0.25 \mathrm{mmol} / \mathrm{L}$ yen $0.30 \mathrm{mmol} / \mathrm{L}$ respectivamente, sin variar la cantidad de $\mathrm{cHDL} 0$ triglicéridos en la sangre ${ }^{2}$, debido a que cuentan con la propiedad de formar soluciones viscosas, lo que impide parcialmente el contacto entre las enzimas digestivas y los nutrientes, tal como los ácidos biliares y los lípidos; este mecanismo disminuye la digestión y absorción de los nutrientes en el intestino delgado, forzando a movilizar glucógeno y lípidos como suministros energéticos, así como para mantener los niveles óptimos de glucosa en la sangre. Igualmente, los ácidos biliares son atrapados y excretados por las heces, obligando a la síntesis de nuevos ácidos biliares a partir del colesterol hepático disponible, lo que explica la reducción del colesterol y los niveles de LDL en sangre ${ }^{13}$.

Kim y colaboradores (2016), también evidenciaron que los $\beta$-glucanos provocan retraso en el vaciamiento gástrico y atenuación de la velocidad de absorción de la glucosa en el intestino delgado debido a que tienen la capacidad de adsorber y retener moléculas orgánicas tal como la glucosa y lípidos ${ }^{25}$.

Por otro lado, Chen y colaboradores (2019), empleando un modelo de ulceración en ratas inducido por etanol, encontraron que el tratamiento con $\beta$-glucanos podría aliviar las lesiones gástricas y el daño de la mucosa gástrica causado por el etanol y por consiguiente reducir el índice de úlcera gástrica. Se demostró que al acrecentar la actividad de superóxido dismutasa y catalasa y disminuyendo el nivel de malondialdehído, los $\beta$-glucanos mitigaron la lesión por estrés oxidativo gástrico en ratas vehículo. También se argumentó que éste podría disminuir el nivel de interleucina- 6 y el factor de necrosis tumoral alfa, al mismo tiempo, aumentar el nivel de prostaglandina E2 y óxido nítrico. Del mismo modo aumentó la longitud del colon y la concentración total de AGCC tanto en colon y ciego de los ratones ${ }^{26}$.

Estudios realizados por Fusté y colaboradores (2019), revelaron que la aplicación tópica de $\beta$-glucanos de cebada $(1 \rightarrow 3)$, $(1 \rightarrow 4)$ mejora la cicatrización de las úlceras diabéticas y venosas. Demostraron que los $\beta$ glucanos de cebada inducen una respuesta temprana de fibroblastos dérmicos humanos, favoreciendo la migración de fibroblastos versus la proliferación, y acelerando el cierre de la herida in vivo ${ }^{27}$. Esto es consistente con el proceso de curación de la herida por el cual los fibroblastos inicialmente migran a la herida, para luego proliferar y producir componentes de la matriz extracelular. La figura 2 muestra imágenes representativas de heridas en diferentes momentos (aproximadamente 12 días después), en 2 grupos de ratones, el grupo tratado con $\beta$-glucanos de cebada en comparación con el grupo control. Donde se puede observar que los $\beta$-glucanos son capaces de mejorar el proceso de curación de heridas in vivo ${ }^{27}$. 


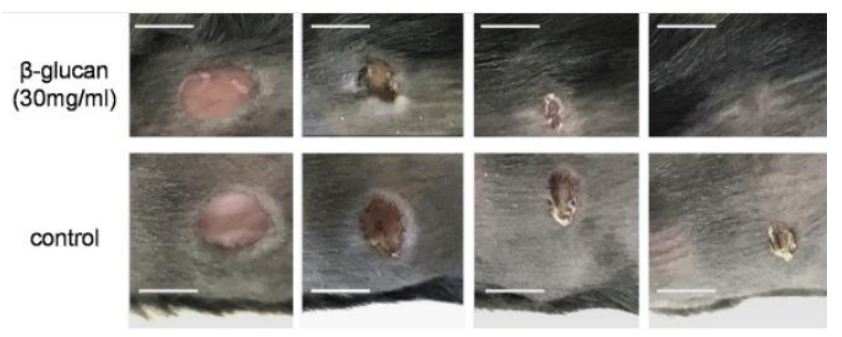

Figura 2. Comparación de heridas, grupo de ratones tratado con $\beta$-glucanos de cebada y grupo control ${ }^{27}$.

Un análisis realizado en el año 2019, reportó que los $\beta$ glucanos orales inducen un cambio sacarolítico en la microbiota que eleva los niveles séricos de AGCC, y del mismo modo reduce los niveles circulantes de $p$-cresilsulfato, la cual es una toxina urémica que compromete la función renal y promueve lesiones cardiovasculares ${ }^{28}$.

Recientemente se emplearon nanopartículas biogénicas de óxido de zinc conjugadas con $\beta$-D-glucanos de cebada para mejorar la actividad anticancerosa y el carcinoma de pulmón, donde se encontró que la apoptosis temprana o tardía era mayor en las células tratadas con nanopartículas de T- $\beta$-D-glu-ZnO (6.43\%) en comparación con las nanopartículas de $\mathrm{T}-\mathrm{ZnO}$ (4\%). Las nanopartículas de T-ZnO y de T- $\beta$-D-glu-ZnO indujeron la muerte de las células cancerosas a través de la vía de necrosis y apoptosis. De igual modo se emplearon nanopartículas de T- $\beta$-D-glu-ZnO en lombrices intestinales, dónde se observó que el tratamiento inhibió significativamente el crecimiento del Staphylococcus aureus dentro de la lombriz intestinal infectada por el patógeno bacteriano, provocando un mayor crecimiento de la lombriz, en comparación con una lombriz intestinal sana $^{29}$.

Se examinaron los efectos de la cebada con alto contenido de $\beta$-glucanos en la obesidad visceral en individuos japoneses. Se evidenció que la cebada rica en $\beta$-glucanos puede contribuir a prevenir la obesidad visceral puesto que la ingesta de cebada con alto contenido de $\beta$-glucanos condujo a reducciones significativas y seguras en el área de grasa visceral, peso corporal, índice de masa corporal y circunferencia de cintura en individuos con área de grasa visceral $\geq 100 \mathrm{~cm}^{2}$ 30

Otro estudio realizado por Xiao y colaboradores (2019), en el que resaltaron los efectos de la fermentación por Lactobacillus plantarum dy-1, se recalcó que la fermentación puede mejorar las actividades fisiológicas in vitro de $\beta$-glucanos de cebada, enfocándose especialmente en el metabolismo de la glucosa y de lípidos. Del mismo modo se pudo apreciar una reducción en la absorción de glucosa y de colesterol en condiciones ácidas resultado de la inhibición de la actividad de $\alpha$ - amilasa, $\alpha$-glucosidasa y lipasa, comparada con los $\beta$ glucanos nativos de cebada ${ }^{31}$.

\section{Aplicaciones tecnológicas}

Se han encontrado múltiples aplicaciones tecnológicas de $\beta$-glucanos en los alimentos. Con el paso de los años se han realizado diversas evaluaciones funcionales para poder conocer y aprovechar de mejor manera sus propiedades tanto funcionales como tecnológicas. Algunas investigaciones han demostrado que los $\beta$ glucanos se pueden aplicar en variados productos de panificación como en harina para bocadillos, galletas, panes, entre otros productos, para mejorar la funcionabilidad de estos. Zhai y colaboradores (2020) examinaron los $\beta$-glucanos de cebada, hallando que reducen la emulsión de lípidos y la digestión independientemente de la viscosidad. Igualmente se detectó que promueve el agotamiento de la floculación, el cual consiste en un proceso de adición de partículas, en el que se forma una baja cantidad de grandes flóculos a través de un gran número de partículas pequeñas de las emulsiones lipídicas $^{32}$. En 2019, Messia y colaboradores analizaron harinas de cebada con un alto contenido de $\beta$ glucanos empleadas para la producción de cuscús funcional, que también es conocido como pasta marroquí. El cuscús funcional presentó atributos saludables a causa de la presencia de $\beta$-glucanos y fibra ${ }^{33}$. Lo que también coincide con el estudio realizado por Lotfi y colaboradores (2019) en bocadillos con harina de cebada y $10 \%$ de orujo de zanahoria mediante cocción por extrusión (cocción a niveles bajos de humedad, comparado con el horneado convencional o la cocción de masas y pastas), a una temperatura de troquel de $142.7^{\circ} \mathrm{C}$ y $14.02 \%$ de contenido de humedad, se pudo notar que la FD soluble aumentó sin modificar el contenido total de FD, por lo que se concluyó que los bocadillos extruidos tenían un alto valor nutricional ${ }^{34}$.

Así también, Gangopadhyay y colaboradores (2019) analizaron 4 tipos de galletas horneadas adicionadas con diferentes fracciones molidas de cebada, evidenciando que pueden servir como ingredientes para mejorar los componentes saludables de las galletas adicionadas 0 sus productos. Se demostró que el salvado de cebada mejora el contenido de $\beta$-glucanos, los polifenoles y la capacidad antioxidante de las galletas ${ }^{35}$.

Djurle y colaboradores (2018), llevaron a cabo una evaluación en panes de cebada, determinando los efectos del horneado sobre la FD, haciendo hincapié en $\beta$-glucanos y almidón resistente. Se observó un incremento de la extractibilidad de $\beta$-glucanos con enlace mixto $(1 \rightarrow 3)$, $(1 \rightarrow 4)$, con la cocción, sin embargo, su peso molecular promedio disminuyó ${ }^{36}$. 
Por otra parte, Zhang y colaboradores (2019), realizaron un estudio en vino de cebada sin cáscara proveniente de zonas altas de China, el cual es una bebida popular de esas zonas. Con el antecedente de que su consumo aporta beneficios para la salud, se determinó el contenido total de $\beta$-glucanos, fenoles y flavonoides totales, demostrando que era abundante tanto en $\beta$-glucanos como en compuestos fenólicos ${ }^{37}$.

Otro estudio realizado por Kaur y Riar (2019) en producto lácteo fermentado (yogur), con adición de extracto de $\beta$ glucanos aislados de cebada con una pureza de $91.52 \%$ $(p \leq 0.05)$ en distintas concentraciones, actuó como un ingrediente bioactivo funcional. Durante el almacenamiento se pudo apreciar un efecto significativo en la sinéresis de suero, viscosidad, perfil de textura y características sensoriales. Las características del análisis de perfiles de textura, como la dureza, la cohesión y la resistencia de las muestras de yogur, también presentaron mejorías ${ }^{38}$.

\section{Conclusión}

El consumo de $\beta$-glucanos de cebada tiene beneficios en la salud, lo que depende de sus propiedades para formar soluciones viscosas en el intestino delgado y su fermentabilidad en colon, así como de la dosis y peso molecular. Por otro lado, las nuevas evidencias sobre sus propiedades cicatrizantes en el tratamiento de úlcera gástrica, diabética y venosa, la reducción del daño renal y cardiovascular, el efecto anticancerígeno y bactericida, abren un amplio campo en la investigación de este compuesto, esto a la par con el desarrollo de alimentos que conserven sus propiedades bioactivas.

\section{Referencias}

[1] Brownlee IA, Chater PI, Pearson JP and Wilcox MD. (2017). Dietary fibre and weight loss: Where are we now? Food Hydrocoll. 68:186191.

[2] Whitehead, A., Beck, E. J., Tosh, S., \& Wolever, T. M. (2014). Cholesterol-lowering effects of oat $\beta$-glucan: a meta-analysis of randomized controlled trials. The American journal of clinical nutrition, 100(6), 1413-1421

[3] Villacrés E, Campaña D, Garófalo J, Falconí E, Quelal M, Matanguihan J and Murphy K. (2019). Evaluation of $\beta$-glucan content, viscosity, soluble dietary fiber and processing effect in grains of Ecuadorian barley genotypes. Agronomía Colombiana 37(3):323-330.

[4] Aktas-Akyildiz E, Sibakov J, Nappa M, Hytönen E, Koksel H and Poutanen K. (2018). Extraction of soluble $\beta$-glucan from oat and barley fractions: Process efficiency and dispersion stability. Journal of Cereal Science, 81:60-68.

[5] De Paula R, Abdel-Aal ESM, Messia MC, Rabalski I and Marconi E. (2017). Effect of processing on the beta-glucan physicochemical properties in barley and semolina pasta. Journal of Cereal Science, 75:124-131.

[6] Kim H and Kim H. (2017). Physicochemical characteristics and in vitro bile acid binding and starch digestion of $\beta$-glucans extracted from different varieties of Jeju barley. Food Sci Biotechnol, 26:1501-1510.

[7] Revuelta I. (2020). 3 Museos. Historia de la cerveza en México. Recuperado el 11 de junio de 2020 de https://www.3museos.com/?eventos=historia-de-la-cerveza-enmexico\#: :text=Hace\%20casi\%20quinientos $\% 20 \mathrm{a} \% \mathrm{C} 3 \% \mathrm{~B} 1$ os $\% 2 \mathrm{C}$ $\% 20$ Alfonso,primer\%20productor\%20de\%20cerveza\%20en

[8] CIMA. Reporte del mercado de cebada, enero 2020. Disponible en: https://www.cima.aserca.gob.mx/work/models/cima/pdf/cadena/2020 /Reporte_mercado_cebada_100120.pdf

[9] Sánchez AR, Martín FM, Palma MS, López PB, Bermejo LLM and Gómez C C. (2015). Indicaciones de diferentes tipos de fibra en distintas patologías. Nutrición Hospitalaria, 31(6):2372-2383.

[10] Aune D, Sen A, Norat T and Riboli E. (2019). Dietary fibre intake and the risk of diverticular disease: a systematic review and meta-analysis of prospective studies. European Journal of Nutrition, 1-12.

[11] Agüero JAR, Palacios CL, Hernández MER, Tobías HMR and Cuello CM. (2019). Polisacáridos estructurales y fibra dietética en brotes florales (Tunitas) de Nopalea cochenillifera (L.) Salm-Dick de diferente estadio de desarrollo. Agrociencia, 53(4):605-616.

[12] Dai FJ and Chau CF. (2017). Classification and regulatory perspectives of dietary fiber. Journal of Food and Drug Analysis, 25(1):37-42.

[13] Vilcanqui-Pérez F and Vílchez-Perales C. (2017). Fibra dietaria: nuevas definiciones, propiedades funcionales y beneficios para la salud. Revisión. Archivos Latinoamericanos de Nutrición, 67(2):146156.

[14] Wong J, De Souza R, Kendall C, Emam A and Jenkins D. (2006). Colonic health: fermentation and short chain fatty acids. Journal of Clinical Gastroenterology, 40(3):235-243.

[15] De Arcangelis E and Djurle S, Andersson AAM, Marconi E, Messia MC and Andersson R. (2019). Structure analysis of $\beta$-glucan in barley and effects of wheat $\beta$-glucanase. Journal of Cereal Science, 85:175181.

[16] Pizarro CS, Ronco MAM and Gotteland RM. (2014). $\beta$-glucanos: ¿qué tipos existen y cuáles son sus beneficios en la salud? Revista Chilena de Nutrición, 41(4):439-446

[17] Kant L, Amrapali, S and Babu BK. (2016). Barley. In Singh M and Upadhyaya HD (ed) Genetic and Genomic Resources for Grain Cereals Improvement, Academic Press, p. 125-157.

[18] Grando S and Macpherson HG. (2005). Food barley: importance, uses and local knowledge. ICARDA, Aleppo, Syria, 121-137.

[19] Baik BK and Ullrich SE. (2008). Barley for food: characteristics, improvement, and renewed interest. Journal of Cereal Science, 48(2):233-242.

[20] Secretaría de Agricultura y Desarrollo Rural. (02 de agosto de 2019). Producción de cerveza en México: una historia para celebrar. Recuperado el 10 de junio de 2020 de https://www.gob.mx/agricultura/articulos/produccion-de-cerveza-enmexico-una-historia-para-celebrar 
[21] Karimi R, Azizi MH and Xu Q. (2019). Effect of different enzymatic extractions on molecular weight distribution, rheological and microstructural properties of barley bran $\beta$-glucan. International Journal of Biological Macromolecules, 126:298-309.

[22] Li Z, Dong Y, Xiao X and Zhou X-H. (2019). Mechanism by which $\beta$-glucanase improves the quality of fermented barley flour-based food products. Food Chemistry, 311:126026.

[23] Danilova TV, Friebe B, Gill BS, Poland J and Jackson E. (2017) Development of a complete set of wheat-barley group-7 Robertsonian translocation chromosomes conferring an increased content of $\beta$ glucan. Theoretical and Applied Genetics, 131(2):377-388.

[24] Zhao Y, Zhou HM, Huang ZH and Zhao RY. (2020). Different Aggregation States of Barley $\beta$-Glucan Molecules Affects Their Solution Behavior: A Comparative Analysis. Food Hydrocolloids, 101:105543.

[25] Kim EK, Oh TJ, Kim LK and Cho YM. (2016). Improving effect of the acute administration of dietary fiber-enriched cereals on blood glucose levels and gut hormone secretion. Journal of Korean Medical Science. 31(29):222-230.

[26] Chen H, Nie Q, Xie M, Yao H, Zhang K, Yin J and Nie S. (2019) Protective effects of $\beta$-glucan isolated from highland barley on ethanol-induced gastric damage in rats and its benefits to mice gut conditions. Food Research International, 122:157-166.

[27] Fusté N, Guasch M, Guillen P, Anerillas C, Cemeli T, Pedraza N, et al. (2019). Barley $\beta$-glucan accelerates wound healing by favoring migration versus proliferation of human dermal fibroblasts Carbohydrate Polymers, 210:389-398.

[28] Arcidiacono MV, Carrillo-López N, Panizo S, Castro-Grattoni AL, Valcheva P, Ulloa C and Dusso AS. (2019). Barley-ß-glucans reduce systemic inflammation, renal injury and aortic calcification through ADAM17 and neutral-sphingomyelinase 2 inhibition. Scientific Reports, 9(1):1-14.

[29] Saravanakumar K, Jeevithan E, Hu X, Chelliah R, Oh DH and Wang M.-H. (2020). Enhanced anti-lung carcinoma and anti-biofilm activity of fungal molecules mediated biogenic zinc oxide nanoparticles conjugated with $\beta$-D-glucan from barley. Journal of Photochemistry and Photobiology B: Biology, 203:111728.

[30] Aoe S, Ichinose Y, Kohyama N, Komae K, Takahashi A, Abe D and Yanagisawa T. (2017). Effects of high $\beta$-glucan barley on visceral fat obesity in Japanese individuals: A randomized, double-blind study. Nutrition, 42:1-6.

[31] Xiao X, Tan C, Sun X, Zhao Y, Zhang J, Zhu Y and Zhou X. (2019) Effects of fermentation on structural characteristics and in vitro physiological activities of barley $\beta$-glucan. Carbohydrate Polymers, 231:115685.x

[32] Zhai H, Gunness P and Gidley MJ. (2020). Barley $\beta$-glucan effects on emulsification and in vitro lipolysis of canola oil are modulated by molecular size, mixing method, and emulsifier type. Food Hydrocolloids, 103:105643.

[33] Messia MC, Oriente M, Angelicola M, De Arcangelis E, and Marconi E. (2019). Development of functional couscous enriched in barley $\beta$ glucans. Journal of Cereal Science 85:137-142.

[34] Lotfi SS, Koocheki A, Milani E, and Mohebbi M. (2020). Production of high fiber ready-to-eat expanded snack from barley flour and carrot pomace using extrusion cooking technology. Journal of Food Science and Technology, 57:2169-2181.
[35] Gangopadhyay N, O'Shea N, Brunton NP, Gallagher E, Harrison SM and Rai DK. (2019). Fate of beta-glucan, polyphenols and lipophilic compounds in baked crackers fortified with different barley-milled fractions. LWT, 114:108413.

[36] Djurle S, Andersson AAM and Andersson R. (2018). Effects of baking on dietary fibre, with emphasis on $\beta$-glucan and resistant starch, in barley breads. Journal of Cereal Science, 79:449-455.

[37] Zhang K, Yang J, Qiao Z, Cao X, Luo Q, Zhao J, Wang F and Zhang W. (2019). Assessment of $\beta$-glucans, phenols, flavor and volatile profiles of hulless barley wine originating from highland areas of China. Food Chemistry, 293:32-40.

[38] Kaur R and Riar CS. (2019). Sensory, rheological and chemical characteristics during storage of set type full fat yoghurt fortified with barley $\beta$-glucan. Journal of Food Science and Technology. 57:41-51. 